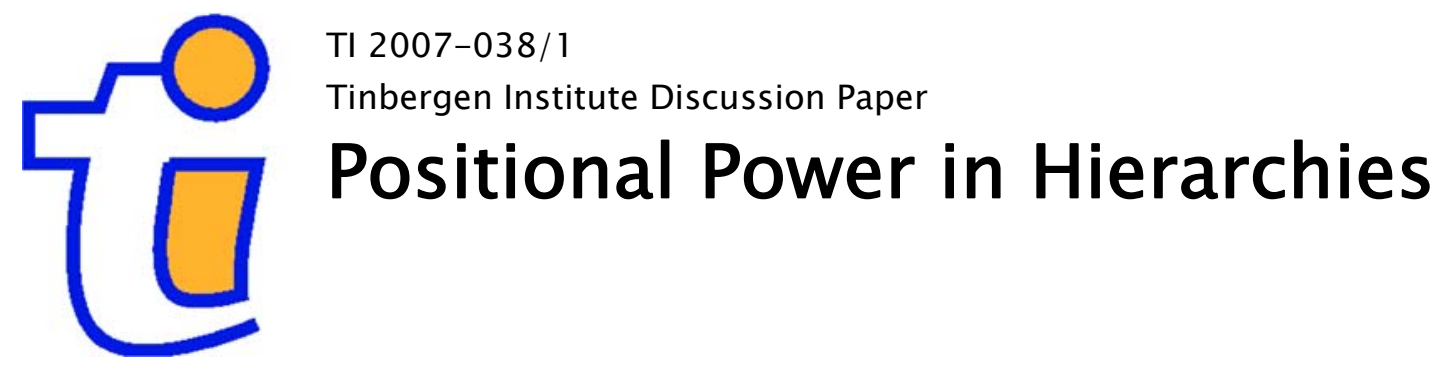

René van den Brink ${ }^{7}$

Frank Steffen ${ }^{2}$

' Dept. of Econometrics, Free University Amsterdam, and Tinbergen Institute;

2 University of Liverpool, Management School (ULMS). 


\section{Tinbergen Institute}

The Tinbergen Institute is the institute for economic research of the Erasmus Universiteit Rotterdam, Universiteit van Amsterdam, and Vrije Universiteit Amsterdam.

Tinbergen Institute Amsterdam

Roetersstraat 31

1018 WB Amsterdam

The Netherlands

Tel.: $\quad+31(0) 205513500$

Fax: $\quad+31(0) 205513555$

Tinbergen Institute Rotterdam

Burg. Oudlaan 50

3062 PA Rotterdam

The Netherlands

Tel.: $\quad+31(0) 104088900$

Fax: $\quad+31(0) 104089031$

Most TI discussion papers can be downloaded at http:/ /www.tinbergen.nl. 


\title{
Positional Power in Hierarchies
}

\section{René van den Brink}

Department of Econometrics and Tinbergen Institute

Free University Amsterdam

jbrink@feweb.vu.nl

\author{
Frank Steffen \\ University of Liverpool \\ Management School (ULMS) \\ steffen2@liv.ac.uk
}

\begin{abstract}
Power is a core concept in the analysis and design of organisations. In this paper we consider positional power in hierarchies. One of the problems with the extant literature on positional power in hierarchies is that it is mainly restricted to the analysis of power in terms of the bare positions of the actors. While such an analysis informs us about the authority structure within an organisation, it ignores the decision-making mechanisms completely. The few studies which take into account the decision-making mechanisms make all use of adaptations of well-established approaches for the analysis of power in non-hierarchical organisations such as the Banzhaf measure; and thus they are all based on the structure of a simple game, i.e. they are 'membershipbased'. We demonstrate that such an approach is in general inappropriate for characterizing power in hierarchies as it cannot be extended to a class of decision-making mechanisms which allow certain actors to terminate a decision before all other members have been involved. As this kind of sequential decision-making mechanism turns out to be particularly relevant for hierarchies, we suggest an action-based approach - represented by an extensive game form - which can take the features of such mechanisms into account. Based on this approach we introduce a power score and measure that can be applied to ascribe positional power to actors in sequential decision making mechanisms.
\end{abstract}

JEL Classification C79, D02, D71

Keywords hierarchies, decision-making mechanism, power, positional power, power measure 



\title{
Positional Power in Hierarchies
}

\author{
René van den Brink \\ Department of Econometrics and Tinbergen Institute, Free University Amsterdam, \\ The Netherlands
}

Frank Steffen

University of Liverpool Management School, U.K.

\section{Introduction}

Power is a core concept for the analysis and design of organisations. The literature contains a wide variety of contributions from various disciplines dealing with different types and aspects of power in organisations. These can broadly be classified by a combination of two features: the subject of the analysis and its primary origin (Morriss 1987/2002: 107f). The subject is either the possession of power or its exercise and its primary origin is either an individual or a position in an organisation. That is, power is taken to be rooted in an individual or a position.

Based on these two features, the literature can, in very general terms, be said to consist of four categories: studies on (i) positional power, (ii) individual power, (iii) the exercise of positional power, and (iv) the exercise of individual power. This paper is devoted to the study of the first category. Positional power is what results from the interplay of two components of an organization's architecture: the arrangement of positions in the organization and the decision-making mechanisms in use.

It is well recognized that the organisational architecture and its resulting power structure are essential ingredients for the success of an organisation (Brickley et al. 2004; Daudi 1986; Johnston and Gill 1993; Martin 1998). Although there exists plenty of work addressing the positional power relations in organisational architectures, ${ }^{1}$ very little attention has been given to investigating such power relations in hierarchical organisations i.e. where there are dominance relations among the actors. This would be unremarkable if hierarchies would not play a significant role in social life. But the op-

\footnotetext{
${ }^{1}$ See, for instance, Felsenthal and Machover (1998), Holler and Owen (2000, 2001, 2002), Holler et al. (2002), Holler and Gambarelli (2006), and the references therein.
} 
posite is true: hierarchies can be found in all areas of social life.

One of the problems with most of the extant literature on positional power in hierarchies is that it is restricted to the analysis of power only in terms of the arrangement of the actors. ${ }^{2}$ While such an analysis informs us about the authority structure within an organisation, it ignores the decisionmaking mechanisms completely.

To the best of our knowledge, only a handful of studies on positional power in hierarchies take into account the decision-making mechanisms (van den Brink 1994, 1997, 1999, 2001; Gilles et al. 1992; Gilles and Owen 1994; van den Brink and Gilles 1996; Berg and Paroush 1998; Shapley and Palamara 2000a,b; and Steffen 2002). All these studies make use of adaptations of well-established approaches for the analysis of power in non-hierarchical organisations such as the Banzhaf (1965) measure; and thus they are all based on the structure of a simple game, i.e. they are 'membership-based'. Roughly speaking, in such a set-up power is ascribed to an actor $i$, if $i$ has the potential to alter an outcome that has been forced by some members of the organisation, by either leaving or joining the subset of actors which has effected the present outcome. In the course of our analysis we will demonstrate that such an approach is in general inappropriate for characterizing power in hierarchies.

To deal with the problem of how to characterize power relations in a hierarchy, we develop an action-based approach based on an extensive game form. Here power is ascribed to an actor $i$, if $i$ has the potential to alter an outcome forced by the members of the organisation by altering his action. A similar action-based approach has been already suggested for decisionmaking mechanisms in non-hierarchical set-ups (Miller 1982), but has so far received only little attention in the literature as it was regarded as an equivalent representation of the membership-based approach. Our analysis will demonstrate that this equivalence only holds for a certain class of decisionmaking mechanisms and that the membership-based approach in contrast to the action-based cannot be extended to a class of decision-making mechanisms which allow certain actors to terminate a decision on behalf of the hierarchy before all other members have been involved. This kind of decision-making mechanism is particularly relevant for hierarchies.

The contribution of our paper can be summarized as follows: (i) We argue that the existing membership-based approaches for the analysis of positional power in hierarchies are only relevant for a (less important) subclass of decision-making mechanisms in hierarchies. (ii) We show that an adequate consideration of the more relevant mechanisms requires an actionbased approach represented by an extensive game form. (iii) We extend the

\footnotetext{
${ }^{2}$ See, for instance, Copeland (1951), Russett (1968), Grofman and Owen (1982), Daudi (1986), Brams (1968), van den Brink (1994, 2002), van den Brink and Gilles (2000), Mizruchi and Potts (1998), Hu and Shapley (2003), Herings et al. (2005), and the references therein.
} 
existing action-based approach which has been formulated for strategic games forms to extensive game forms. (iv) We illustrate that the existing membership-based approaches can be represented as special cases of the action-based approach.

\section{Directed Graphs}

Given that we will represent both the description of the arrangements of the actors and decision-making mechanisms in hierarchical organisations by directed graphs - the two are not necessarily identical - we start by briefly recalling some definitions of such graphs. A directed graph (or digraph) is a set of objects, called nodes joined by directed links called arcs.

Formally, a digraph is an ordered pair $(\mathscr{V}, \mathscr{D})$, where $\mathscr{V}$ is a finite set of nodes (or vertices) of the graph and $\mathscr{D}$ is a set of ordered pairs of $\mathscr{V}$ called arcs of the graph, i.e. $\mathscr{D} \subseteq \mathscr{V} \times \mathscr{V}$ is a binary relation on $\mathscr{V}$. An arc $(i, j) \in \mathscr{D}$ is considered to be directed from node $i$ to node $j$ where $i$ is called a predecessor of $j$ and $j$ is called a successor of $i$ in $\mathscr{R}$. By $\mathscr{S}(i)$ and $\mathscr{S}^{-1}(i)$, respectively, we denote the set of all successors and predecessors of $i \in \mathscr{V}$ in $\mathscr{D}$; i.e. $\mathscr{S}(i)=\{j \in \mathscr{V} \mid(i, j) \in \mathscr{D}\}$ and $\mathscr{S}^{-1}(i)=\{j \in \mathscr{V} \mid(j, i) \in \mathscr{D}\}$. A node $i$ is called a terminal node, if no arc is starting from it, i.e. $\mathscr{S}(i)=\varnothing$.

If $(i, j) \notin \mathscr{D}$, we say that there exists a path from $i$ to $j$ denoted by $\mathscr{P}(i, j)=_{\text {def }} i \rightarrow \ldots \rightarrow j$, if $i \neq j$ and $j$ can be reached from $i$ by following arcs of the graph, e.g. if $(i, h),(h, j) \in \mathscr{D}$, then $\mathscr{P}(i, j)=i \rightarrow h \rightarrow j$. If such a path $\mathscr{P}(i, j)$ exists, we call $i$ and $h$ ancestors of $j$, and $h$ and $j$ descendants of $i$. Moreover, the set of all nodes being descendants of a node $i$ is denoted by $\hat{\mathscr{S}}(i)$ and the set of all ancestors of $i$ by $\hat{\mathscr{S}}^{-1}(i)$. If, instead, $(i, j) \in \mathscr{D}$, we say that $\mathscr{P}(i, j)$ denotes a direct path, and if $(i, j) \notin \mathscr{D}$, but $i=j$, we say that $\mathscr{P}(i, j)$ denotes a degenerated path.

A directed graph is said to be a tree $\mathscr{T}$ if (i) there exists a distinguished node $r \in \mathscr{V}$ (the root of the tree) that has no arcs going into it, and (ii) for every other node $i \in \mathscr{V} \backslash\{r\}$ there exists exactly one path from $r$ to $i$. Furthermore, a tree $\mathscr{T}$ becomes a labelled tree denoted by the triple $\left(\mathscr{T}, l^{\mathscr{V}}, l^{\mathscr{T}}\right)$, if there exist labelling functions $l^{\mathscr{V}}: \mathscr{V} \rightarrow \mathscr{L}^{\mathscr{V}}$ and $l^{\mathscr{D}}: \mathscr{D} \rightarrow \mathscr{L}^{\mathscr{P}}$ with $\mathscr{L}^{\mathscr{V}}$ and $\mathscr{L}^{\mathscr{D}}$ being the corresponding sets of labels.

Moreover, a branch of a tree $\mathscr{T}$ is a directed graph having nodes starting at a node $i \in \mathscr{V}$ and containing all its descendents together with their original arcs. By $\mathscr{T}_{i}$ we denote the branch starting at $i$. Thus, $\mathscr{T}_{i}$ is itself a tree, the root of which is $i$.

\section{Hierarchies}

Hierarchies form a certain subclass of organisational architectures. Following van den Brink (1994) they distinguish themselves from other architectures by the arrangement of its members being connected via directed relations, 
which we interpret as dominance (or superior to) relations. Loosely speaking, we can say that an actor $i$ in a dominating position has an influence on the 'powers' of other actors who are in positions that are dominated by $i$. Domination can be either indirect or direct, i.e. with or without intermediate actors, respectively. Note, that if we just make use of the term 'domination' without further specification, we allow for indirect and direct domination.

Actors in dominating positions are called superiors (or principals) - bosses or managers in common parlance -, while the actors in dominated positions are called subordinates (or agents). If we refer to a superior who directly dominates another actor, the dominating actor is called a predecessor, and if we refer to a subordinate who is directly dominated by another actor, the dominated actor is called a successor.

Formally, we can represent the dominance structure of a hierarchy as a digraph, where the nodes $j \in \mathscr{V}$ represent the actors $i \in N$, i.e. $l^{\mathscr{V}}: \mathscr{V} \rightarrow N$, and the arcs indicate direct dominance relations between the actors, i.e. if there is an arc $(i, j) \in \mathscr{D}$ predecessor $i$ dominates successor $j$ and that $j$ is dominated by $i$. Moreover, we say $\forall j \in \hat{\mathscr{S}}(i)$ that $j$ is a subordinate of $i$, and $\forall j \in \hat{\mathscr{S}}^{-1}(i)$ that $j$ is a superior of $i$.

The dominance relations in a hierarchy are assumed to fulfil the following three properties (see Radner 1992 for a similar set of properties):

Property 3.1 (Transitivity) If $i$ dominates $j$, and $j$ dominates $k$, then $i$ dominates $k$.

Property 3.2 (Anti-symmetry) If $i$ dominates $j$, then $j$ cannot dominate $i$.

Note that by Property (3.1) in combination with (3.2) we assume $\forall i \in N: i \notin \hat{\mathscr{S}}(i)$, i.e. we exclude the case that a dominance structure is cyclic.

Property 3.3 (Single Topness) There is exactly one actor, called the top (or root), who is in a position such that he dominates all other actors. Except for the top, every other actor has at least one predecessor. Thus, formally, there exists an $i \in N$ such that $\hat{\mathscr{S}}(i)=N \backslash\{i\}$ and $\hat{\mathscr{S}}^{-1}(i)=\varnothing$.

For an illustration Figure 1 depicts all feasible dominance structures of hierarchies with two and three members (except structures with relabelled members) which fulfil properties (3.1)-(3.3). Formally, for Figure 1a these are given by $\mathscr{S}(a)=\{b\}$ and $\mathscr{S}(b)=\varnothing$, for Figure 1 b by $\mathscr{S}(a)=\{b\}$, $\mathscr{S}(b)=\{c\}$ and $\mathscr{S}(c)=\varnothing$, and for Figure $1 \mathrm{c}$ by $\mathscr{S}(a)=\{b, c\}$ and $\mathscr{S}(b)=\mathscr{S}(c)=\varnothing$.

Note that the dominance relations in a hierarchy cannot always be represented by a tree, i.e. it is not necessarily the case that except for the top each other actor has one and only one predecessor. In other words, dominance 


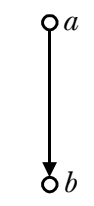

Fig. 1a

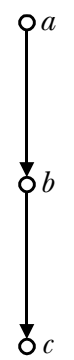

Fig. $1 b$

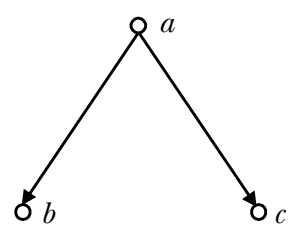

Fig. 1c

Fig. 1. Feasible dominance structures with two and three actors

relations which can be represented by a tree form a subclass of all feasible sets of dominance relations fulfilling properties (3.1)-(3.3).

As a dominance structure is only a partially ordered set, not all actors in a hierarchy are necessarily comparable in terms of their bare dominance relations. For instance, in Figure $1 c, b$ does not dominate $c$, nor does $c$ dominate $b$ (Radner 1992: 1391). However, in everyday language, the word hierarchy not only connotes an upside-down-tree-like dominance structure, but also an assignment of rank which allows for a certain mode of comparison of the positions of $b$ and $c$. By a ranking of a dominance structure we shall mean an assignment of a natural number called the rank (or level) to each actor, such that:

Property 3.4 $i$ has a higher rank (larger number) than $j$, if $i$ dominates $j$.

Property 3.5 $i$ and $j$ have the same rank, if $i$ does not dominate $j$ nor $j$ dominates $i$, i.e. if $i$ and $j$ are not comparable in terms of the dominance relations, and the length of the longest path to the top is the same for both.

While properties (3.4) and (3.5) are sufficient for existence of a ranking, they are only necessary but not sufficient for a unique ranking. Throughout this paper we will make use of a unique ranking which additionally satisfies the following properties: ${ }^{3}$

Property 3.6 There is only one actor with the highest rank $k$ called the top.

Property 3.7 Recursively, an actor has rank $l \leq k-1$ iff all his predecessors have a rank $h \geq l+1$ and at least one of his predecessors has rank $l+1$.

\footnotetext{
${ }^{3}$ Note, that with these properties we exclude hierarchies which contain direct dominance relations over more than one rank (Radner 1992: 1391). While we impose this restriction to keep the exposition of our paper traceable, this does not imply that our approach is not capable to take such hierarchies into account.
} 
Property 3.8 We fix $k$ such that the lowest ranked actor has rank 1 .

An actor is said to be at the bottom (or a leaf) if he does not dominate any other position, i.e. if he owns a terminal node. Note that this does not imply that such a position necessarily belongs to the lowest rank 1.

\section{Decision-Making Mechanisms in Hierarchies}

Decision-making mechanisms are the other component of an organisation's architecture that we need to examine. For the definition of a decision-making mechanism let us start with the general definition of a decision:

Definition 4.1 A decision is the choice of a non-empty proper sub-set of elements out of a set of elements.

In our case the sets of elements from which an actor can choose is created by proposals submitted to the organisation. The chosen (or selected) elements are outcomes produced by members of an organisation. The choices are made by one or more actors belonging to the organisation where each of these actors has to perform an action to make his individual choice effective. How those actions and the outcomes are linked is given by the decision rule:

Definition 4.2 A decision rule is a function which maps ordered sets of individual actions into outcomes.

Thus, a decision rule is an action guiding norm which states which ordered sets of actions generate which outcome. ${ }^{4}$ A second component we require for our definition of a decision-making mechanism is that of a decision-making procedure:

Definition 4.3 A decision-making procedure provides the course of actions of the actors for a collective decision and states which set of actions are counted, i.e. which actions go into the domain of the decision rule.

Now we can define a decision-making mechanism:

Definition 4.4 A decision rule together with a decision-making procedure establishes a decision-making mechanism (DMM).

\footnotetext{
${ }^{4}$ To put it into the language of causality: a decision rule defines the set of all necessary and sufficient conditions for each outcome, i.e. for each outcome it defines a set of sets of actions where each set of actions is sufficient for that outcome.
} 
The canonical set-up for a DMM $\tau$ in a hierarchy as found in the power literature is characterized by the following set of assumptions:

Assumption 4.5 Proposals submitted to the hierarchy are exogenous: it is the task of the hierarchy either to accept or to reject the proposal, i.e. we have a binary outcome set $\mathscr{O}=\{$ acceptance, rejection $\}$.

Assumption 4.6 A proposal can be submitted to the hierarchy only once.

Assumption 4.7 A hierarchy contains a finite set of members $N=$ $\{a, b, \ldots, n\}$ whose actions bring about the decision of the hierarchy.

Assumption 4.8 Each actor $i \in N$ has a binary action set $A_{i}=\{y e s, n o\}$ where yes means that $i$ supports a proposal and no that $i$ rejects the proposal.

Assumptions (4.5)-(4.8) are also common in the analysis of power in non-hierarchical organisations. In addition for hierarchies we need to assume:

Assumption 4.9 The direction of the decision-making procedure through the hierarchy is bottom-up.

Assumption 4.10 New proposals can only be received by those actors $\tilde{N} \subseteq N$ who have a contact to the outside world, i.e. to actors $i \notin N$ submitting the proposals.

Assumption 4.11 Only certain subsets of $\tilde{N}$ can receive a new proposal at the same time. The set of such feasible subsets is given by $\mathscr{N} \subseteq 2^{\hat{N}}$.

Assumption 4.12 If $N^{\prime} \in \mathscr{N}$ receives a new proposal its members have to choose their individual actions. Depending on the DMM, these actions either establish a final decision on behalf of the hierarchy or they imply that the proposal is forwarded to the next higher rank in the hierarchy. Here $\hat{S}^{-1}\left(N^{\prime}\right)$ defines the set of all superiors who may be involved in the decision about that proposal received by $N^{\prime}$ until a final decision on behalf of the hierarchy is made.

Assumption 4.13 If a proposal is forwarded to the next higher rank, there is a set of minimal feasible sub-sets of superiors $\hat{\mathscr{S}}_{\min }^{-1}\left(N^{\prime}\right) \subseteq \hat{\mathscr{S}}^{-1}\left(N^{\prime}\right)$ whose actions are required for the decision about a new proposal that has been presented to $N^{\prime}$.

Assumptions (4.9)-(4.13) require some justification. Let us begin with the very basic rationale of a hierarchy: why may it be useful to have a hierar- 
chy. Why do we have organisations with dominance structures? A common answer to this question says that hierarchies are cost saving because dominance structures allow for the decentralisation of decision-making (delegation), i.e. by dividing tasks between the members of the different ranks and positions. In our context delegation means that we can avoid that all members of an organisation are always involved in all decisions. Hence, DMMs in hierarchies imply that in certain instances particular actors are intentionally excluded from decisions (see, for instance, Mackenzie 1976: 103). This is usually achieved by a combination of two basic principles: (i) by reducing the breadth of the hierarchy involved in a particular decision and (ii) by allowing non-top actors to make certain types of final decisions on behalf of the whole hierarchy even before one of their superiors has been involved. It would therefore be natural, that a generic DMM for a hierarchy should take those principles into account.

To investigate whether such a generic DMM also satisfies assumptions (4.9)-(4.13) we need to determine if there exists a reasonable example based on the very simple hierarchy given by Figure 1c that fulfils both principles (i) and (ii) and the assumptions.

Example 4.14 Assume that a hierarchy with a dominance structure as given by Figure 1c is a development agency with $a$ being its head and $b$ and $c$ being representatives in different developing countries. Moreover, assume that $b$ and $c$ receive funding applications for development projects, i.e. they have a contact to the outside world, while $a$ does not. Suppose that the decision-making on these applications is delegated to $b$ and $c$ according to the above two principles. Based on principle (i) each representative is only responsible for the projects in his country, i.e. is not involved in decisions for the other country. If applications arrive at the desk of one of the representatives $(b$ or $c$ ), this representative decides on them only together with $a$. Furthermore, based on principle (ii), if a representative ( $b$ or $c$ ) receives an application, he is entitled to reject it without contacting $a$ (he can exclude $a$ from the decision-making in this particular instance) although for an approval he requires the consent of $a .{ }^{5}$

Thus, due to principle (i) a decision will never require the whole breath of the hierarchy ( $b$ and $c$ together) to take part in a decision which implies that never all three actors are involved in a decision. In terms of its DMM the hierarchy is truncated twice, i.e. for each actor with a contact to the outside world we obtain a truncated hierarchy containing this actor and all his superiors. ${ }^{6}$ To put it into other words: we have an ensemble of possible games

\footnotetext{
${ }^{5}$ For an overview of further DMMs that can be derived from the literature on positional power in hierarchies see the appendix.

${ }^{6}$ Formally this phenomenon can also taken as abstention, although in this case it is decreed rather than voluntary.
} 
on $N$ with overlapping sets of actors, i.e. $N_{1}=\{a, b\}$ and $N_{2}=\{a, c\} .{ }^{7}$ Whether $b$ or $c$ will be excluded from the decision-making (which possible game will be played) depends on who receives the new proposal. Moreover, due to principle (ii) all non-top actors are entitled to make a certain type of final decision on behalf of the whole hierarchy. This also means that principle (ii) implies a sequential decision-making procedure as it entitles nontop actors to terminate the collective decision-making, before actors on higher ranks are involved.

Now let us determine if Example (4.14) and, thus, the application of the above principles, match with assumptions (4.9)-(4.13). Obviously, Assumption 4.9 is fulfilled. Moreover, the same applies to the remaining assumptions: $\tilde{N}=\{b, c\}(4.10), \mathscr{N}=\{\{b\},\{c\}\}(4.11), \hat{\mathscr{S}}^{-1}(\{b\})=\hat{\mathscr{S}}^{-1}(\{c\})=\{a\}$ (4.12), and $\hat{\mathscr{S}}_{\min }^{-1}(\{b\})=\hat{\mathscr{S}}_{\min }^{-1}(\{c\})=\{a\}$ (4.13). However, note that these assumptions do not take into account that principle (ii) implies a sequential decision-making procedure. An essential characteristic of DMMs in hierarchies is overlooked.

\section{Decision-Making Mechanisms and Extensive Game Forms}

For modelling a DMM in a hierarchy the extant literature applies the classical membership-based approach. However, this is inappropriate because it does not allow for the exclusion of agents based on principle (ii) nor does it allow for the bottom-up structure of the decision-making procedure (Assumption 4.9). Both exclusion and bottom-up decision-making require a sequential structure while the membership-based approach is inherently simultaneous (see Section 7). Hence, we suggest the natural method is an action-based approach that makes use of an extensive game form (EGF) as defined below.

Let $N=\{1, \ldots, n\}$ be the set of actors of a collective decision making body and $N^{*}=_{\text {def }} N \cup \theta$ where $\theta$ denotes 'nature' which behaves randomly, i.e. $\theta$ 's behaviour represents the exogenous effects of the outside world on $N$. The actors are part of a tree of a game form. This is a labelled tree $\left(\mathscr{T}, l^{\mathscr{V}}, l^{\mathscr{P}}\right)$ where (i) $l^{\mathscr{V}}$ assigns to each non-terminal node $j \in \mathscr{V}$ exactly one $i \in N^{*}$ and to each terminal node $k \in \mathscr{V}$ an outcome $a(k) \in \mathscr{O}$ with $\mathscr{O}$ being a non-empty finite outcome set, and (ii) $l^{\mathscr{D}}$ assigns to each arc a move $a \in A$ where $A$ denotes the set of all possible moves for all $i \in N^{*}$.

\footnotetext{
7 The idea of a possible game has been introduced in the context of abstention (see Braham and Steffen 2002). Assume a DMM under which abstention is permissible for any actor $i \in N$. Hence, a decision can be made by any subset $N_{k} \subseteq 2^{N}$. Here any game played on $N_{k}$ is called a possible game on $N$. Note that the idea of a possible game is different to that of a composed game (Shapley 1962). In case of a composed game all components of the game can be played at the same time, while out of the set of possible games only one will be played.
} 
In a tree of a game form a non-terminal node is called a decision-making node if it is owned by an actor $i \in N$, and a chance node if it is owned by $\theta$. Moreover, we denote the set of nodes owned by an actor $i$ by $\mathscr{V}_{i}$.

An information set for an actor $i$ is a set of nodes $h_{i}$ such that in a decision-making procedure $i$ knows that he must make the 'next decision', at some node $j \in h_{i}$, but due to his lack of information of the 'history' of the decision-making procedure $i$ does not know at which node $j \in h_{i}$ exactly he must make his decision; $i$ only knows that $j \in h_{i}$. Necessary conditions of an information set for an actor $i$ are that (i) all nodes of $h_{i}$ are owned by $i$; (ii) no node of $h_{i}$ is related to any other node of $h_{i}$, i.e. if $j, h \in h_{i}$, then $j$ is neither an ancestor nor a descendant of $h$; and (iii) all nodes of $h_{i}$ are equivalent for $i$ with respect to the outgoing arcs, i.e. the number and labelling of arcs starting from each node $j \in h_{i}$ is the same. Moreover, let us denote the set of all information sets of an actor $i$ by $\mathscr{H}_{i}$, i.e. $\mathscr{H}_{i}={ }_{\text {def }}\left\{h_{i 1}, . ., h_{i p}\right\}$. Note, that if the set of nodes of a tree of a game form is $N^{*}$, i.e. it includes $\theta$, we follow the convention that nodes belonging to nature are always elements of singleton information sets

Now we can characterise an EGF.

Definition 5.1 An EGF is a tree of a game form such that the decisionnodes have been partitioned into information sets that belong to the actors.

In an EGF we call $\mathscr{C}_{i}: \mathscr{V}_{i} \rightarrow 2^{\mathscr{V}}$ the choice function of an actor $i \in N^{*}$, if $\mathscr{C}_{i}(j)=\mathscr{S}(j)$ for all $j \in \mathscr{V}_{i}$. Thus, a choice function assigns to each $j \in \mathscr{V}_{i}$ its corresponding set of successors $\mathscr{S}(j)$ in the tree of the game form (it assigns to each $j \in \mathscr{V}_{i}$ the nodes that $i$ can reach being in $j$ and making a move).

Furthermore, we say that $i$ 'has chosen', if $i$ has to make a move in a node $j \in \mathscr{V}_{i}$ and has decided for a move to a node $h \in \mathscr{S}(j)$. For all $i \in N$ we call such choices of a move an action of $i$ in $j$, denoted by $a_{i}^{j}$. The set of all actions available to $i$ at a node $j \in \mathscr{V}_{i}$ is called the action set of $i$ at $j$, denoted by $A_{i}^{j}$, i.e. $A_{i}^{j}={ }_{\text {def }}\left\{a_{i 1}^{j}, \ldots, a_{i m}^{j}\right\}$. The set of all actions available to $i$ at any node in $\mathscr{V}_{i}$ is denoted by $A_{i}$, i.e. $A_{i}=\operatorname{def}_{\text {de }} \cup\left\{j \in \mathscr{V}_{i}: A_{i}^{j}\right\}$.

If instead nature $\theta$ moves, it's moves - if they are non-fictitious - are not actions of intention, but just moves (determining which 'real' actor has to choose next) which follow a probability distribution resulting from a function $p: \mathscr{H}_{\theta} \times A_{\theta} \rightarrow[0,1]$ that assigns probabilities to the 'actions' at information sets where nature moves, satisfying $\forall h_{\theta} \in \mathscr{H}_{\theta}$ : $\sum\left\{a_{\theta}^{j} \in A_{\theta}^{j} \mid j \in h_{\theta}: p\left(a_{\theta}^{j}\right)\right\}=1$.

Next, let us say that for an $i \in N^{*}, \quad j \in \mathscr{V}_{i}$. and $a_{i}^{j} \in A_{i}^{j}$ in an EGF, $h\left(j, a_{i}^{j}\right)$ denotes that node $h \in \mathscr{S}(j)$ such that $l^{\mathscr{P}}(j, h)=a_{i}^{j}$, i.e. $h\left(j, a_{i}^{j}\right)$ is

\footnotetext{
${ }^{8}$ For more details about extensive game forms we refer the reader to Kolpin $(1988,1989)$ and for extensive form games to Kuhn (1953) and Selten (1975).
} 
Table 1. Choice Functions and Related Action Sets

\begin{tabular}{ccc}
\hline$j \in \mathscr{V}_{i}$ & $\mathscr{S}(j)$ & $A_{i}^{j}$ \\
\hline$j \in \mathscr{V}_{a}$ & $\{$ acceptance, rejection $\}$ & $\{$ yes, no $\}$ \\
$j \in \mathscr{V}_{b}$ & $\{a$, rejection $\}$ & $\{$ yes, no $\}$ \\
$j \in \mathscr{V}_{c}$ & $\{a$, rejection $\}$ & $\{$ yes, no $\}$ \\
$j \in \mathscr{V}_{\theta}$ & $\{b, c\}$ & - \\
\hline
\end{tabular}

Table 2. Action Profiles and Related Outcomes

\begin{tabular}{|c|c|}
\hline$a \in \mathscr{A}$ & $a \in \mathscr{O}$ \\
\hline ( $b$ is allowed to choose, yes $s_{b}$ yes ${ }_{a}$ ) & acceptance \\
\hline ( $c$ is allowed to choose, yes $s_{c}$ yes $)$ & acceptance \\
\hline ( $b$ is allowed to choose, yes ${ }_{b}, n_{a}$ ) & rejection \\
\hline ( $c$ is allowed to choose, yes no $_{a}$ ) & rejection \\
\hline ( $b$ is allowed to choose, $n o_{b}$ ) & rejection \\
\hline ( $c$ is allowed to choose, $n o_{c}$ ) & rejection \\
\hline
\end{tabular}

that node that is reached from node $j$, if $i$ makes the move $a_{i}^{j}$. Based on this notation we can define an action profile in an EGF:

Definition 5.2 An 'action profile' $a$ in an EGF is an ordered set of individual moves, $\boldsymbol{a}={ }_{\text {def }}\left(a^{j_{1}}, \ldots, a^{j_{p}}\right)$, belonging to a subset of actors $N(\boldsymbol{a}) \subseteq N^{*}$, such that their moves form a path $\mathscr{P}\left(j_{1}, j_{p+1}\right)=j_{1} \rightarrow j_{2} \rightarrow \ldots \rightarrow j_{p+1}$ with $\mathscr{S}^{-1}\left(j_{1}\right)=\varnothing, \mathscr{S}\left(j_{p+1}\right)=\varnothing$, and $l^{\mathscr{D}}\left(j_{q}, j_{q+1}\right)=a^{j_{q}}$ for all $q \in\{1, \ldots, p\}$.

Thus, an action profile $\boldsymbol{a}$ is a 'path of moves' within the tree of the game form where the first move begins at the root $r=j_{1}$ and the last move ends at a terminal node $k=j_{p+1}$. The set of all action profiles will be denoted by $\mathscr{A}$ and by $\mathscr{A}_{i} \subset \mathscr{A}$ we will denote the subset of all action profiles that contain an action of actor $i$.

Notice that the action profiles in an EGF which represents a DMM consider only counted individual actions and not all feasible individual actions. That is, these action profiles ignore those actions which actors have available and can perform but which are not counted by the decision-making procedure.

Let us return to Example (4.14) which can now be represented by an EGF with $N^{*}=\{a, b, c, \theta\}, \quad \forall j \in \mathscr{V}_{i}, i \in N: A_{i}^{j}=\{$ yes, no $\}, \mathscr{O}=\{$ acceptance, 


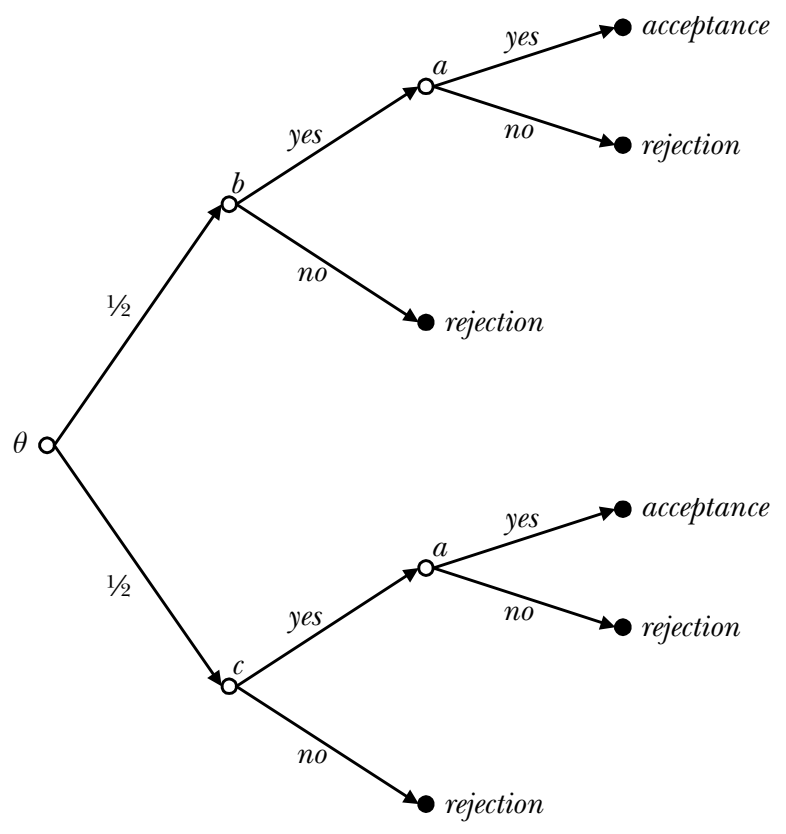

Fig. 2. The DMM as an EGF

rejection $\}, \quad \forall i \in N: \# \mathscr{H}_{i}=\# \mathscr{V}_{i}$, and, thus, $\forall j \in \mathscr{V}_{i}:\{j\} \in \mathscr{H}_{i}$. Hence, we have an EGF with perfect information, i.e. the information sets are singletons and there is one information set $h_{i}$ for each node $j$ owned by $i{ }^{9}$ Given the triple $\left(N^{*}, \tau, \mathscr{S}\right)$ with $\tau$ denoting the DMM as described by Example (4.14) we obtain the choice functions and the individual action sets as given by Table 1 . From these we can derive the action profiles containing the counted individual actions and their related outcomes as given by Table 2 .

What is left to be specified is the probability function determining nature's moves, $p$. It determines which actors with a contact to the outside world will obtain a proposal on their desk and, therefore, establishes which possible game will be played. In the absence of any (structural) information regarding the likelihood of $N^{\prime} \in \mathscr{N}$ to receive a new proposal, we apply the principle of insufficient reason of classical probability theory. ${ }^{10}$ This assigns equal probability to all admissible 'atomic events', i.e. in the present case equal probability to all $N^{\prime} \in \mathscr{N}$ for receiving a new proposal:

\footnotetext{
${ }^{9}$ Note, that in a hierarchy an actor may own more than one decision node, even if he owns only one node in the dominance structure.

${ }^{10}$ In the absence of any information about the outside world the application of the principle of insufficient reason appears to be legitimate here as we fulfil the condition that we have a finite probability space consisting of finitely many clearly distinguished indivisible 'atomic events' (Felsenthal et al. 2003).
} 


$$
p\left(N^{\prime} \text { receives a new proposal }\right)=1 / \# \mathscr{N}
$$

which implies that for each actor $i \in \tilde{N}$ his probability to receive a new proposal is given by

$$
\hat{p}(i \in \tilde{N} \text { receives a new proposal })=\#\left\{N^{\prime} \in \mathscr{N} \mid i \in N^{\prime}\right\} / \# \mathscr{N} \text {. }
$$

We can now give a proper graphical representation of a DMM as an EGF. For Example (4.14) this is given by Figure 2. From Section 4 we know that we have two possible games with $N_{1}=\{a, b\}$ and $N_{2}=\{a, c\}$. Moreover, we have $\mathscr{N}=\{\{b\},\{c\}\}$ which implies that each possible game occurs with probability $\frac{1}{2}$. In the EGF this is reflected by the assignment of this probability to the moves of nature which lead to the branches $\mathscr{T}_{b}$ and $\mathscr{T}_{c}$ representing the possible games.

\section{Measuring Positional Power: An Action-Based Approach}

Our understanding of 'power' is based on Harré (1970) and Morriss $(1987 / 2002)$ who define power as a concept that always refers to a generic (and therefore, in a sense, timeless) ability or capacity of an object. In a social context this object is an actor and a power ascription refers to his ability: what the actor is able to do against the resistance of at least some other actor. Following Braham (2007) we say that an actor $i$ has power with respect to a certain outcome if $i$ has an action (or sequence of actions) such that the performance of the action under the stated or implied conditions will result in that outcome despite the actual or possible resistance of at least some other actor. That is, power is a claim about what $i$ is able to do against some resistance of others irrespective of the actual occurrence of the resistance. Thus, power is a capacity or potential which exists whether it is exercised or not. In our context, this capacity is based on the positions of the actors in an organisation.

The measurement of power involves the following steps: (i) The identification of the action profiles within the organisation that are sufficient for bringing about an outcome. (ii) The ascription of power to an individual actor in these action profiles by determining if the actor has an action that if performed will, ceteris paribus, alter the outcome of the collective action. (iii) The aggregation of the individual power ascriptions of each actor, giving us a bare power score. (iv) The weighting of the aggregated power ascriptions yields to a power measure. If this weighting is such that all aggregated power ascriptions sum up to unity we say that we have a power index. The difference between a score and a measure rests in the comparability of power structures: a score allows for ordinal comparisons only, while a measure allows for cardinal comparisons. 
In Section 5 we already described and illustrated step (i) (see Table 2). Thus, we can immediately proceed with step (ii). To ascribe power to an actor we examine for each action profile $a$ whether $i$ has an action, ${ }^{11}$ which, if chosen, can make a decisive difference to the outcome against the actual or possible resistance of at least some other actor. That is, we determine if a given actor $i$ has a swing.

To define a swing in an EGF let $\hat{\mathscr{O}}(g)=_{d e f}\{a \in \mathscr{O} \mid$ there is a path $\mathscr{P}(g, k)$ with $\mathscr{S}(k)=\varnothing$ and $\varrho=a(k)\}$, i.e. $\hat{\mathscr{O}}(g)$ is a relation between a decision node $g \in \mathscr{V}_{i}$ and the outcome set $\hat{\mathscr{O}}$ that can be achieved from this node. ${ }^{12}$ Based on this relation we have:

Definition 6.1 A swing of an actor $i$ is a triple $\left(a, a_{i}^{j_{l}}, \tilde{a}_{i}^{j_{l}}\right)$ with $\boldsymbol{a}=\left(a^{j_{1}}, \ldots, a^{j_{p}}\right)$ being an action profile and actions $a_{i}^{j_{l}}, \tilde{a}_{i}^{j_{l}} \in A_{i}^{i_{l}}$ for which there are two decision nodes $j_{l} \in \mathscr{V}_{i}$ and $j_{k} \in \mathscr{S}\left(j_{l}\right)$ that are reached by profile $a$ and a decision node $g \in \mathscr{S}\left(j_{l}\right) \backslash\left\{j_{k}\right\}$, such that $l^{\mathscr{P}}\left(j_{l}, j_{k}\right)=a_{i}^{j_{l}}$, $l^{\mathscr{Q}}\left(j_{l}, g\right)=\tilde{a}_{i}^{j_{l}}$, and $\hat{\mathscr{O}}(g) \backslash\left\{a\left(a^{j_{p}}\right)\right\} \neq \varnothing$.

Hence, $i$ has a swing, if $i$ can alter the resulting outcome $o\left(a^{j_{p}}\right)$ of an action profile $a$ by, ceteris paribus, changing his action $a_{i}^{j l}$.

At this point it is important to draw attention to the interpretation of the ceteris paribus condition. In the context of collective decision-making it is commonly said to imply that the actions of all other actors remain constant (in all information sets). That is, if $i$ alters his action the only effect that can result out of this is a change in the outcome (then we say that $i$ has a swing and we ascribe power to $i$ ). While this 'all other things being equal' interpretation is appropriate for a simultaneous DMM, it no longer applies for our more general case of a sequential DMM, which may allow certain actors to exclude other actors from the decision-making as a result of their choices (see principle (ii) in Section 4). If we have an action profile $a \in \mathscr{A}_{i}$ and we alter $i$ 's action $a_{i}^{j t}$ in $a$ it can happen that the decision-making process requires either the exclusion of actions of other actors $a_{i^{\prime}}^{j_{1}}$ from the domain of the decision rule and, hence, from action profile $a$, or the inclusion of actions by other actors $\tilde{a}_{i^{\prime} j^{\prime}}$ in the domain of the decision rule and, therefore, in action profile $a$. If such information would be ignored, we can end up with an inappropriate power ascription resulting out of the alteration of $i$ 's action.

In order to avoid this problem and to capture this information we have to go back to the idea behind the literal 'all other things being equal' interpretation of the ceteris paribus clause. The basic idea of the ceteris paribus clause is a comparison between two possible worlds: the world as it is (our

\footnotetext{
11 The literature on power commonly uses the term 'strategy' as a synonym for an 'action' (see Miller 1982; Braham and Steffen 2003; Braham and Holler 2005) even if power is defined as the ability of an actor to effect outcomes by his chosen actions and not by his plan of action.

${ }^{12}$ Note, that we allow $\mathscr{P}(g, k)$ to be a direct path, but not to be a degenerated path.
} 
initial action profile $a$ and its associated outcome $\left.a\left(a^{j_{p}}\right)\right)$ and the world as it would be if an action were changed (the resulting action profile and its associated outcome if $i$ 's action $a_{i}^{j_{l}}$ were altered). In contrast to the standard interpretation of the ceteris paribus clause our analysis does not necessarily require that all other components of the action profile remain constant after we altered $i$ 's action; it requires that the action profiles after the initial change by one actor are consistent with the DMM. Hence, this interpretation of the ceteris paribus clause which is underlying Definition (6.1) is able to capture the above mentioned aspects of a sequential DMM. ${ }^{13}$

If an actor $i$ has a swing, we can distinguish between two different types of swings:

Definition 6.2 A swing $\left(a, a_{i}^{j l}, \tilde{a}_{i}^{j l}\right)$ of an actor $i$ is strong if $\# \hat{\mathscr{O}}(g)=1$, and weak if $\# \hat{\mathscr{O}}(g)>1$, with $g$ as given in Definition 6.1.

Note, that for a swing $\left(a, a_{i}^{j_{l}}, \tilde{a}_{i}^{j_{l}}\right)$ and $g$ as given in Definition 6.1, $\# \hat{\mathscr{O}}(g)=1$ implies that the unique outcome in $\hat{\mathscr{O}}(g)$ is different from the outcome $a\left(a^{j p}\right)$ of the action profile $a$ since by definition of a swing $\hat{\mathscr{O}}(g)$ contains at least one outcome that is different than $a\left(a^{j_{p}}\right)$. Thus, a strong swing enables an actor to alter a unique outcome into another unique outcome, while a weak swing only enables an actor to alter a unique outcome into a non-unique outcome.

The distinction between strong and weak swings is novel and becomes necessary due to the sequential structure of our approach. Both swings are immediately comparable if we have set inclusion, i.e. a strong swing $\left(\boldsymbol{a}, a_{i}^{j l}, \tilde{a}_{i}^{j l}\right)$ and a weak swing $\left(\boldsymbol{a}^{\prime}, a_{i}^{j{ }^{j \prime}}, \tilde{a}_{i}^{j \prime \prime}\right)$ with $g$ and $g^{\prime}$, respectively, as given in Definition (6.1) are comparable if $\hat{\mathscr{O}}(g) \subset \hat{O}^{\prime}\left(g^{\prime}\right)$. In this case which applies to our binary set-up, a strong swing implies more power (or ability) than a weak swing as the outcome that an actor with a strong swing can enforce is more specific. Moreover, for a binary set-up the literature distinguishes between positive and negative swings:

Definition 6.3 For a DMM represented by an EGF with $\forall j \in \mathscr{V} i$, $i \in N: A_{i j}=\{y e s, n o\}$ and $\mathscr{O}=\{$ acceptance,rejection $\}$ an actor $i$ has a positive swing, if $i$ by switching from a ' $n o$ '- to a 'yes'-action can alter the outcome from a 'rejection' to an 'acceptance' and has a negative swing, if $i$ by switching from a 'yes'- to a ' $n o$ '-action can alter the outcome from an 'acceptance' to a 'rejection'.

\footnotetext{
${ }^{13}$ Note that the ceteris paribus clause in Definition (6.1) also guarantees the fulfillment of the resistance condition (see Braham 2007).
} 
Definition 6.4 Let $S_{i}=\left\{s_{i}^{1}, \ldots, s_{i}^{z}\right\}$ be the set of all swings of an actor $i$ where each $s_{i}$ denotes a swing $\left(\boldsymbol{a}, a_{i}^{j_{l}}, \tilde{a}_{i}^{j l}\right)$, Moreover, let $S_{i}^{s}$ and $S_{i}^{w}$ denote the sets of strong and weak swings of $i$ respectively.

For step (iii), the individual power ascriptions for each actor given by $S_{i}$ are aggregated in order to obtain a complete and transitive relation 'at least as powerful as for $a^{\prime}, \succcurlyeq_{a}$, defined over $N{ }^{14}$

To aggregate the individual power ascriptions we have to take into account the existence of nature, or in other words: the potential exclusion of actors from the decision-making due to principle (i) (exclusion due to principle (ii) is already taken into account via the action profiles). We obtain this by weighting each swing on a branch $\mathscr{T}_{j}$ with $j \in \mathscr{V}_{i}$ and $i \in \tilde{N}$ with the likelihood that nature will choose this branch. For the EGF this implies that $\exists j \in \mathscr{V}_{\theta}: \mathscr{S}^{-1}(j)=\varnothing$, i.e. that nature owns the root $r$ of the tree of the game form, and, hence, makes the first move. Furthermore, for our analysis we assume $\# \mathscr{V}_{\theta}=1$, i.e. that nature owns only one node being the root. ${ }^{15}$

Definition 6.5 Let $p\left(a_{\theta} \in a\right)$ be the likelihood that nature chooses the move $a_{\theta}$ that is part of the action profile $\boldsymbol{a}$, and $\boldsymbol{a}\left(s_{i}\right)$ be the action profile in a swing $s_{i}=\left(\boldsymbol{a}, a_{i}^{j_{l}}, \tilde{a}_{i}^{j_{l}}\right)$. Then $\mu\left(a_{\theta} \in \boldsymbol{a}\left(s_{i}\right)\right)$ denotes the likelihood that nature chooses the move that is part of the action profile $a$ in swing $s_{i}$.

Note: (i) If in the (original) EGF $\exists j \in \mathscr{V}_{\theta}: \mathscr{S}^{-1}(j)=\varnothing, \# \mathscr{V}_{\theta}=1$, and $\# N^{\prime}=1$ for all $N^{\prime} \in \mathscr{N}$, due to the principle of insufficient reason we have $\mu\left(a_{\theta} \in \boldsymbol{a}\right)=p\left(N^{\prime}\right.$ receives a new proposal $)=1 / \# \mathscr{N}$. (ii) If in the (original) EGF $\mathscr{V}_{\theta}=\varnothing$, i.e. if nature does not exist, we can represent this EGF by a strategically equivalent EGF with $\exists j \in \mathscr{V}_{\theta}: \mathscr{S}^{-1}(j)=\varnothing, \quad \# \mathscr{V}_{\theta}=1$, and $\# A_{\theta}=1$, which implies \# $\mathscr{N}=1$, and, hence, $\mu\left(a_{\theta} \in a\right)=p\left(N^{\prime}\right.$ receives a new proposal) $=1$.

Definition 6.6 The power score of an actor $i \in N$ in a decision-making situation represented by an EGF is given by $\eta_{i}\left(N^{*}, \tau, \mathscr{S}\right)={ }_{\text {def }} \sum\left\{s_{i} \in S_{i}^{s}\right.$ :

\footnotetext{
${ }^{14}$ Note, that here $\forall i, j \in N: i \succcurlyeq_{0} j$ is to be interpreted as ' $i$ 's degree of power to force $\theta$ is at least as great as $j$ 's degree of power to force $\sigma^{\prime}$, with $\succ_{a}$ and $\sim_{a}$ denoting the asymmetric and symmetric components of $\succcurlyeq_{a}$, i.e. $\succ_{a}$ denotes 'the greater degree of power' and $\sim_{0}$ 'the same degree of power'.

${ }^{15}$ Although this assumption might appear to be quite strong, it does not impose any restriction on the applicability of our approach to DMMs represented by EGFs. Naturally, it can happen that for certain DMMs $\exists j \in \mathscr{V}_{\theta}: \mathscr{S}^{-1}(j) \neq \varnothing$, i.e. that nature owns also other nodes than the root. However, in these cases, it is easy to proof via a backward induction procedure, that we can represent the original EGF by a strategically equivalent one where we have merged the nodes $j \in \mathscr{V}_{\theta}$, such that $\# \mathscr{V}_{\theta}=1$ and $\mathscr{S}^{-1}(j)=\varnothing$ for $j \in \mathscr{V}_{\theta}$.
} 
$\left.\mu\left(a_{\theta} \in \boldsymbol{a}\left(s_{i}\right)\right)\right\}+\sum\left\{s_{i} \in S_{i}^{w}: \varepsilon \cdot \mu\left(a_{\theta} \in \boldsymbol{a}\left(s_{i}\right)\right)\right\}$ with $0<\varepsilon<1$ to take into account the nature of weak swings. ${ }^{16}$

From Definition (6.1) it follows that in this case $\succcurlyeq_{a}$ is a cardinality-based ranking such that $\forall i, j \in N: i \succcurlyeq_{0} j \rightarrow \eta_{i} \geq \eta_{j}$.

In order to obtain a power measure allowing for cardinal comparisons, we have to continue with step (iv) by applying different weightings to the action profiles and, thus, to the related power ascriptions (to the swings of the actors). From Definition (6.6) we can derive the natural analogue to the Banzhaf measure $\beta^{\prime}$ which is formulated in the language of the membership-based approach (Banzhaf 1965, Felsenthal und Machover 1998). ${ }^{17} \mathrm{We}$ obtain this analogue for an actor $i \in N$ by weighting the score with the weighted sum of 'potential swings' of $i$ in all action profiles $a \in \mathscr{A}_{i}$. Here the weight of each action profile $a$ is determined by the likelihood that nature chooses this profile, i.e. $p\left(a_{\theta} \in a\right)$. The number of potential swings of $i$ in each profile $a$ results out of the sum of alternative actions $\tilde{a}_{i}^{j} \in A_{i}^{j} \backslash\left\{a_{i}^{j}\right\}$ actor $i$ has at hand for each action $a_{i}^{j} \in \boldsymbol{a}$ :

Definition 6.7 The power measure of an actor $i \in N$ in a decision-making situation represented by an EGF is given by

$$
\tilde{\beta}_{i}^{\prime}\left(N^{*}, \tau, \mathscr{S}\right)={ }_{\text {def }} \frac{\sum\left\{s_{i} \in S_{i}^{s}: \mu\left(a_{\theta} \in \boldsymbol{a}\left(s_{i}\right)\right)\right\}+\sum\left\{s_{i} \in S_{i}^{w}: \varepsilon \cdot \mu\left(a_{\theta} \in \boldsymbol{a}\left(s_{i}\right)\right)\right\}}{\sum\left\{\boldsymbol{a} \in \mathscr{A}_{i}: \mu\left(a_{\theta} \in \boldsymbol{a}\right) \sum\left\{j \in \mathscr{V}_{i}(\boldsymbol{a}):\left(\# A_{i}^{j}-1\right)\right\}\right\}}
$$

where $\mathscr{V}_{i}(\boldsymbol{a})$ is the set of decision nodes $j \in \mathscr{V}_{i}$ that are reached by $\boldsymbol{a}$.

Now let us return to Example (4.14). For the analysis of the power structure of this example we start by exploring the actors' swings. From Figure 2 we know that $a$ owns two nodes: one in each possible game, while $b$ and $c$ own one node in 'their' possible game. In each node each actor has two actions. Examining the effects of altering, ceteris paribus, the actions for all

\footnotetext{
${ }^{16}$ Note, that for the binary DMMs which are discussed in the literature (see Appendix) it is not necessary to specify the value of $\varepsilon$ as these DMMs all include (i) bottom-up procedures which (ii) always requires the presence of the top for an approval: (i) ensures that the top is the only one who owns a positive strong swing, while (ii) guarantees that the top is at least never less powerful than any other actor if a dominance structure satisfies properties (3.1)(3.8). If we want to specify the value of $\varepsilon$, we could be tempted to think about an actor dependent operator $\varepsilon_{i}$. However, this would make the power of an actor $i$ additionally dependent on the actions and, thus, the powers of his subsequent actors in the EGF - something which is in contradiction to our dispositional concept of power, i.e. that the power of an actor $i$ is what $i$ is able to do when he is in the position to act.

${ }^{17}$ The derivation of other analogues of well known power measures, such as the ShapleyShubik (1954) index is left to future research.
} 
actors shows that $a$ has a positive and a negative strong swing in each of the two games, whereas $b$ and $c$ only have one positive weak and one negative strong swing each.

Let us have a closer look at $b$ 's situation. If $b$ switches from ' $n o$ ' to 'yes' $b$ can exclude a straightforward rejection against the resistance of all other actors performing any feasible action, and if $b$ switches from a 'yes' to ' $n o$ ', $b$ can ensure a straightforward rejection against the resistance of all other actors performing any feasible action. Furthermore, and as already indicated above, here the ceteris paribus clause for $b$ implies not only that $b$ has an effect on the outcome but also on $a$ 's action: by switching his action $b$ determines whether $a$-according to the DMM - has to act or not and whether $a$ 's action is counted, i.e. if $b$ opts for ' $y e s$ ' $a$ is obliged to act and his action is counted, while if $b$ chooses ' $n o$ ' $a$ is neither obliged to act nor his action is counted. Thus, a switch in $b$ 's action forces us either to consider an action performed by $a$ which was not counted or even not existent before, or to ignore an originally counted action performed by $a$. However, the sole effect of $b$ on $a$ is something which is irrelevant for our analysis which is concerned with the question whether $b$ has the ability to affect the outcome at that decision-making node.

Taking into account that each possible game occurs with equal probability, it is obvious that $a$ is more powerful than $b$ and $c$ and that $b$ and $c$ have equal power, i.e. $a \succ_{0} b \sim_{0} c$. Applying Definition (6.6) and (6.7) we obtain the following power distributions which reflect this ordering:

$$
\begin{aligned}
\eta\left(N^{*}, \tau, \mathscr{S}\right) & =(2.00,0.50+0.50 \varepsilon, 0.50+0.50 \varepsilon) \\
\tilde{\beta}^{\prime}\left(N^{*}, \tau, \mathscr{S}\right) & =(1.00,0.33+0.33 \varepsilon, 0.33+0.33 \varepsilon) .
\end{aligned}
$$

\section{Measuring Positional Power: Pathologies of the Membership- Based Approaches}

We mentioned earlier that the literature on the measurement of positional power in non-hierarchical organisations is usually founded on a membership-based approach. We also stated that the membership-based approach is not suitable to deal with the particularities of DMMs which are typical for hierarchical organisations. To see why, let us start with the canonical set-up of the membership-based approach. This is represented by a simple game.

Definition 7.1 A simple game $(\mathrm{SG})$ is a pair $(N, \mathscr{W})$ where $\mathscr{W}$ is a collection of subsets (coalitions) of the set of actors $N$ called the set of winning coalitions, which satisfies the following three conditions: $\varnothing \notin \mathscr{W} ; N \in \mathscr{W}$; and (monotonicity) if $T \in \mathscr{W}$ and $T \subseteq T^{\prime}$, then $T^{\prime} \in \mathscr{W}$. A subset $T \subseteq N$ is said to be winning or losing according to whether $T \in \mathscr{W}$ or $T \notin \mathscr{W}$. 
Furthermore, a subset $T$ is called minimal winning iff $T \in \mathscr{W}$, but no subset of $T$ is in $\mathscr{W}$. The set of minimal winning subsets is denoted by $\mathscr{M}$.

Hence, a DMM represented by a SG can be either represented by $\mathscr{W}$ or due to the monotonicity condition by $\mathscr{M}$, where a coalition $T$ can be regarded as an 'index' of the actions of the actors which have chosen the same action, for instance, 'yes', if $T \in \mathscr{W}$.

Now, let us investigate the degree to which a SG is able to meet Assumptions (4.5) and (4.7)-(4.13) and both principles discussed in Section 4. This will provide us with an idea about the limitations of this approach and how we can convert the membership-based approach founded on a SG into a special case of our action-based approach.

Like our EGF the definition of a SG is also based on a finite set of actors $N$ (Assumption 4.7). The binary structure of the decision-making situation, i.e. the individual binary action sets (Assumption 4.8) and the binary outcome set (Assumption 4.5) are taken into account via the membership of actors in winning or losing subsets of actors. An actor establishes his membership of such a subset by his individual action, i.e. by either supporting or rejecting a proposal. Thus, what a SG does is that it subdivides all actors subject to their individual actions to belong either to $T$ or $N \backslash T$ where $T$ contains those actors who "get their way" according to the DMM, i.e. whose individual actions corresponds to the collective outcome. ${ }^{18}$ The fact that new proposals can only be received by actors with a contact to the outside world (Assumption 4.10) is taken into account by the requirement that $\forall T \in \mathscr{M}: T \cap \tilde{N} \neq \varnothing$. The assumption that only subsets of $\tilde{N}$ can receive a new proposal at the same time (Assumption 4.11) is currently not taken into account by the existing membership-based approaches but could be incorporated by applying the idea of possible games with overlapping sets of actors. The same applies if a collective decision does not require the whole breath of a hierarchy, i.e. principle (i) (see Example 4.14). The information that in certain instances particular actors $i$ are able to determine the collective decision by their individual action (Assumptions 4.12 and 4.13) can be considered via veto rights, i.e. that $\forall T \in \mathscr{M}: i \in T$.

What remains is the bottom-up direction of the decision-making procedure (Assumption 4.9) and the assumption that particular actors might be able to make certain types of final decisions on behalf of the whole hierarchy even before one of their superiors has been involved (principle ii). Both cannot be taken into account by a SG because they require a sequential structure while a SG is inherently simultaneous.

\footnotetext{
${ }^{18}$ Note, that this additionally requires the usual assumption that the simple game is selfdual, i.e. that any binary division of actors results in exactly one winning and one losing subset (Taylor and Zwicker 1999).
} 


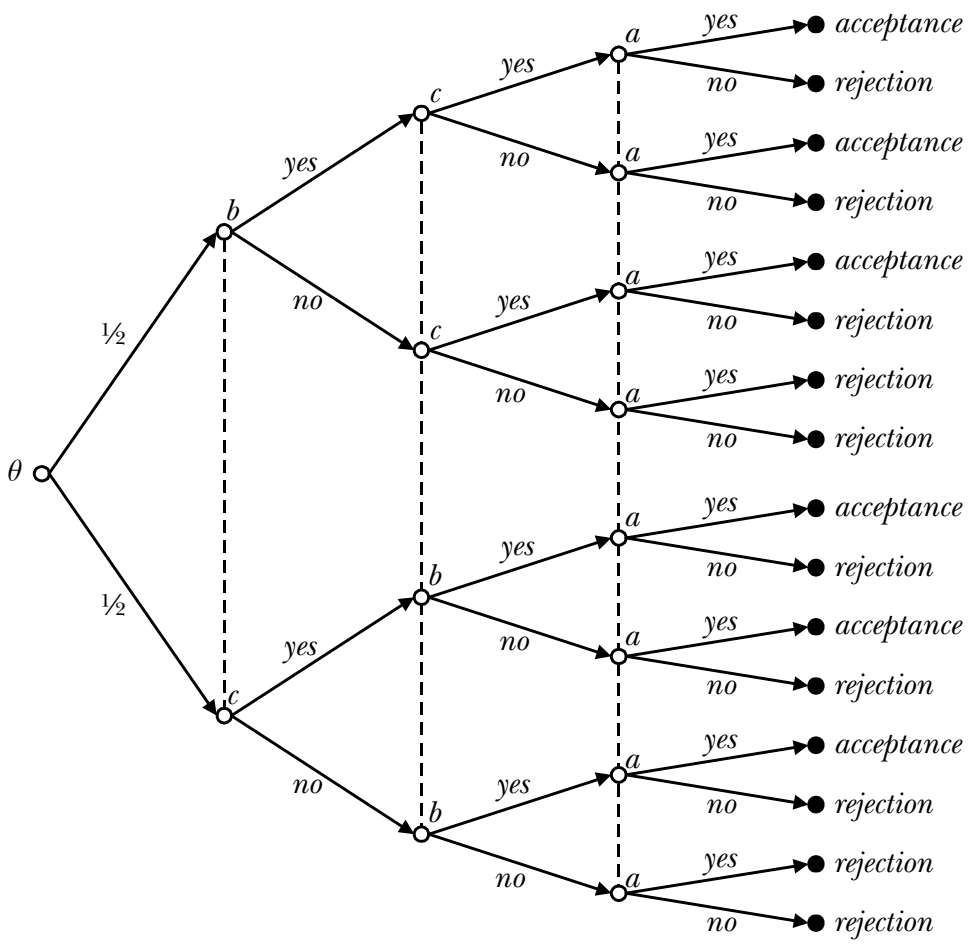

Fig. 3. An EGF representation of a SG

A SG only considers the memberships of actors in $T \in \mathscr{W}$ or $T \notin \mathscr{W}$, but not when the actors join such subsets. Thus, it implicitly assumes that all actors join the subsets at the same time. Hence, the membership-based SG setup can be represented by an EGF with simultaneous individual actions. Put it differently: by an EGF with imperfect information such that there is one information set containing all nodes owned by $i$, i.e. $\forall i \in N: \# \mathscr{H}_{i}=1$ and $h_{i}=\mathscr{V}_{i}{ }^{19}$

Thus, the major difference between our and the existing approaches is that they differ in the assumptions of the information sets. This has farreaching consequences. Not only are the trees of the game form considerably different (see Figure 3 for the EGF of Example 4.14 with imperfect information), but also the power structure.

Altering the information sets of Example (4.14) in the appropriate way we obtain $\eta\left(N^{*}, \tau, \mathscr{S}\right)=(6.00,2.00,2.00)$ and $\tilde{\beta}^{\prime}\left(N^{*}, \tau, \mathscr{S}\right)=(0.75,0.25$, $0.25)$. The ordering is the same, but $a$ becomes relatively more powerful

\footnotetext{
${ }^{19}$ Note, that a DMM represented by a SG can also be represented by a strategic game form (SGF) with unconditional actions (Miller 1982) which implies that a SGF with conditional actions is an alternative of equal rank to the EGF we suggest here.
} 
than $b$ and $c$. This is due to the fact that in a simultaneous decision-making structure - in contrast to a sequential structure - all feasible individual actions are counted. However, the effects of ignoring the sequential structure of a DMM in a hierarchy and instead applying an approach with a simultaneous structure might be stronger than in this example, i.e. it might change the power ordering of the actors.

Example 7.2 Assume a hierarchy with the dominance structure given by Figure 1b. Applying the membership-based set-up we have $N^{*}=N=$ $\{a, b, c\}, \forall j \in \mathscr{V}_{i}, i \in N: A_{i j}=\{y e s, n o\}, \forall i \in N: \# \mathscr{H}_{i}=\# \mathscr{V}_{i}, \quad$ and $\quad \forall j \in \mathscr{V}_{i}:$ $\{j\} \in \mathscr{H}_{i}$, and $\mathscr{O}=\{$ acceptance, rejection $\}$. Concerning the DMM $\tau$ let us assume that only $c$ has contact to the outside world, i.e. $\{\tilde{N}\}=\mathscr{N}=\{\{c\}\}$, that the decision-making procedure is bottom-up, and that each actor is able to reject a proposal on behalf of the whole hierarchy if it arrives at his desk and he rejects it - which implies that subsequent individual actions should not be counted - while an acceptance of a proposal requires the approval of all three actors. Then we obtain $\eta\left(N^{*}, \tau, \mathscr{S}\right)=(2.00,1.00+\varepsilon$, $1.00+\varepsilon$,$) and \tilde{\beta}^{\prime}\left(N^{*}, \tau, \mathscr{S}\right)=(1.00,0.33+0.33 \varepsilon, 0.25+0.25 \varepsilon)$, while in case of $\forall i \in N$ : \# $\mathscr{H}_{i}=1$ and $h_{i}=\mathscr{V}_{i}$, i.e. under a simultaneous structure with $\mathscr{W}=\mathscr{M}=\{\{a, b, c\}\}$, we would obtain $\eta\left(N^{*}, \tau, \mathscr{S}\right)=(2.00,2.00,2.00)$ and $\tilde{\beta}^{\prime}\left(N^{*}, \tau, \mathscr{S}\right)=(0.25,0.250 .25)$. Hence, the power ordering $a \succ_{a} b \succ_{a} c$ based on the power measure taking into account the sequential nature would be misrepresented by $a \sim_{a} b \sim_{a} c$ under the simultaneous structure.

\section{Concluding Remarks}

We would like to tie up this paper with a brief summary of our results and some remarks. We provided an action-based framework for the analysis of positional power in organisational architectures which is able to take into account the particularities of hierarchical structures, For this reason our approach is more general than the usual membership-based approach which does not allow for sequential DMMs. Moreover, we demonstrated that if it is still applied to a sequential DMM it may result in inappropriate cardinal and ordinal power structures as it ascribes power to actors in action profiles which should not be counted.

In order to measure an actors' power we have extended the notion of a swing from a simultaneous to a sequential set-up and aggregated the individual power ascriptions over all action profiles of which an actor is a member. This is in line with our definition of power as a generic ability which is a conditional disposition that exists irrespective whether it is exercised or not.

For the derivation of our power measure in Definition (6.7) from our power score in Definition (6.6) we divided the score of an actor $i$ by the weighted sum of his potential swings. Even though this reflects perfectly what the Banzhaf measure is doing for the simultaneous case, it is not so in- 
nocent as it seems to be - even for our binary set-up. While under the simultaneous structure all actors are members of all action profiles and, hence, each actor has the same chance to have a swing in each action profile, this is no longer necessarily the case under the sequential set-up. A consequence of this is that our power score and measure are not necessarily co-monotone, i.e. Felsenthal et al's (1998) price monotonicity postulate is violated.

Finally, we have to acknowledge that the suggested approach currently does not take into account the case of differing incentive structures between the actors, i.e. that actors have a damatis personae: they are the bearers of predetermined attributes and modes of behaviour. Actors in hierarchies often play predetermined roles, such as salesman, financial officer, head of external affairs, etc., which are equipped with a bundle of incentive structures that also belong to the organisational architecture. Steffen (2002) argues that a proper measurement of power should take such information into account if it is available. For the membership-based approach Straffin's $(1977,1978)$ partial homogeneity approach offers a solution to this problem. Even though we expect that the same idea could be applied to our action-based approach, this, as well as the axiomatization of our measure, are issues for future research.

\section{Appendix}

Based on Assumptions (4.5)-(4.13) we can identify 12 DMMs from the literature on positional power in hierarchies (Table A.1). These differ in the explicit definitions of $\tilde{N}, \mathscr{N}$, and $\hat{\mathscr{S}}_{\min }^{-1}\left(N^{\prime}\right)$ as given by Assumptions (4.10), (4.11), and (4.13) while they all share the other assumptions with $\hat{\mathscr{S}}^{-1}\left(N^{\prime}\right)=\left\{j \in \hat{\mathscr{S}}^{-1}(i) \mid i \in N^{\prime}\right\}$ for Assumption (4.12). Furthermore, note, that $i$ and $j$ denote here actors in a dominance structure and not in an EGF.

Table A.1

\begin{tabular}{ccc}
\hline$\tilde{N}$ & $\mathscr{N}$ & $\hat{\mathscr{S}}_{\min }^{-1}\left(N^{\prime}\right)$ \\
\hline$N$ & $\tilde{N}$ & $\left\{\left\{j \in \hat{\mathscr{S}}^{-1}(i) \mid i \in N^{\prime} \wedge \mathscr{S}^{-1}(j)=\varnothing\right\}\right\}$ \\
$N$ & $\tilde{N}$ & $\left\{\mathscr{P}(i, j) \mid i \in N^{\prime} \wedge j \in \hat{\mathscr{S}}^{-1}(i) \wedge \mathscr{S}^{-1}(j)=\varnothing\right\}$ \\
$N$ & $\tilde{N}$ & $\left\{\left\{j \in \hat{\mathscr{S}}^{-1}(i) \mid i \in N^{\prime}\right\}\right\}$ \\
$N$ & $\left\{N^{\prime} \in 2^{\tilde{N}} \mid \# N^{\prime}=1\right\}$ & $\left\{\left\{j \in \hat{\mathscr{S}}-1(i) \mid i \in N^{\prime} \wedge \mathscr{S}^{-1}(j)=\varnothing\right\}\right\}$ \\
$N$ & $\left\{N^{\prime} \in 2^{\tilde{N}} \mid \# N^{\prime}=1\right\}$ & $\left\{\mathscr{P}(i, j) \mid i \in N^{\prime} \wedge j \in \hat{\mathscr{S}}^{-1}(i) \wedge \mathscr{S}^{-1}(j)=\varnothing\right\}$ \\
$N$ & $\left\{N^{\prime} \in 2^{\tilde{N}} \mid \# N^{\prime}=1\right\}$ & $\left\{\left\{j \in \hat{\mathscr{S}}^{-1}(i) \mid i \in N^{\prime}\right\}\right\}$ \\
\hline
\end{tabular}


... / Table A.1

\begin{tabular}{ccc}
\hline$\tilde{N}$ & $\mathscr{N}$ & $\hat{\mathscr{S}}_{\text {min }}^{-1}\left(N^{\prime}\right)$ \\
\hline$\{i \in N \mid \mathscr{S}(i)=\varnothing\}$ & $\tilde{N}$ & $\left\{\left\{j \in \hat{\mathscr{S}}^{-1}(i) \mid i \in N^{\prime} \wedge \mathscr{S}^{-1}(j)=\varnothing\right\}\right\}$ \\
$\{i \in N \mid \mathscr{S}(i)=\varnothing\}$ & $\tilde{N}$ & $\left\{\mathscr{P}(i, j) \mid i \in N^{\prime} \wedge j \in \hat{\mathscr{S}}^{-1}(i) \wedge \mathscr{S}^{-1}(j)=\varnothing\right\}$ \\
$\{i \in N \mid \mathscr{S}(i)=\varnothing\}$ & $\tilde{N}$ & $\left\{\left\{j \in \hat{\mathscr{S}}^{-1}(i) \mid i \in N^{\prime}\right\}\right\}$ \\
$\{i \in N \mid \mathscr{S}(i)=\varnothing\}$ & $\left\{N^{\prime} \in 2^{\tilde{N}} \mid \# N^{\prime}=1\right\}$ & $\left\{\left\{j \in \hat{\mathscr{S}}^{-1}(i) \mid i \in N^{\prime} \wedge \mathscr{S}^{-1}(j)=\varnothing\right\}\right\}$ \\
$\{i \in N \mid \mathscr{S}(i)=\varnothing\}$ & $\left\{N^{\prime} \in 2^{\tilde{N}} \mid \# N^{\prime}=1\right\}$ & $\left\{\mathscr{P}(i, j) \mid i \in N^{\prime} \wedge j \in \hat{\mathscr{S}}^{-1}(i) \wedge \mathscr{S}^{-1}(j)=\varnothing\right\}$ \\
$\{i \in N \mid \mathscr{S}(i)=\varnothing\}$ & $\left\{N^{\prime} \in 2^{\tilde{N}} \mid \# N^{\prime}=1\right\}$ & $\left\{\left\{j \in \hat{\mathscr{S}}^{-1}(i) \mid i \in N^{\prime}\right\}\right\}$
\end{tabular}

\section{Acknowledgements}

We would like to thank Marlies Ahlert, Steve Brams, Franz Dietrich, Keith Dowding, Bernhard Grofman, Thomas Hammond, Marc Kilgour, Hartmut Kliemt, Martin Leroch, Moshé Machover, Stefan Napel, Jan-Willem van der Rijt, Federico Valenciano, Stefano Vannucci, William Zwicker, and in particular Matthew Braham, Manfred Holler, and Pete Morriss for comments and discussions. Forerunners of this paper have been presented the Meeting of the Dutch Social Choice Group at Tilburg University (January 2006), the 2006 Annual Meeting of the European Public Choice Society in Turku (April 2006), the Eighth International Meeting of the Society for Social Choice and Welfare in Istanbul (July 2006), at the conference on 'Power: Conceptual, Formal, and Applied Dimensions' in Hamburg (August 2006), and at the 2006 Annual Meeting of the American Political Science Association in Philadelphia (August/September 2006). This article was largely written while Frank Steffen at Tilburg University under a Marie Curie Intra-European Fellowship within the 6th European Community Framework Programme. He gratefully acknowledges this financial support.

\section{References}

Banzhaf, J.F. (1965) Weighted Voting Doesn't Work: A Mathematical Analysis, Rutgers Law Review 19: 317-341.

Berg, S. and Paroush, J. (1998), Collective Decision Making in Hierarchies, Mathematical Social Sciences 35: 233-244.

Braham, M. (2007) Social Power and Social Causation: Towards a Formal Synthesis, in M. Braham and F. Steffen (eds), Power: Conceptual, Formal, and Applied Dimensions (provisional title), Springer.

Braham, M. and Holler, M.J. (2005) The Impossibility of a Preference-based Power 
Index, Journal of Theoretical Politics 17: 137-57.

Braham, M. and Steffen, F. (2002) Voting Power in Games with Abstentions, in M.J. Holler et al. (eds), Jahrbuch für Neue Politische Ökonomie 20: Power and Fairness: Mohr Siebeck, 333-348.

Braham, M. and Steffen, F. (2003) Voting Rules in Insolvency Law: A Simple Game Theoretic Approach, International Review of Law and Economics 22: 1-22.

Brams, S. (1968) Measuring the Concentration of Power in Political Systems, American Political Science Review 62: 461-475.

Brickley, J.A., Smith, C.W., and Zimmerman, J.L. (2004) Managerial Economics and Organizational Architecture, McGraw Hill/Irwin.

Brink, R. van den (1994), Relational Power in Hierarchical Organizations, PhD dissertation, Tilburg University.

Brink, R. van den (1997) An Axiomatization of the Disjunctive Permission Value for Games with a Permission Structure, International Journal of Game Theory 26: 27-43.

Brink, R. van den (1999) An Axiomatization of the Conjunctive Permission Value for Games with a Hierarchical Permission Structure, in H. de Swart (ed.), Logic, Game Theory and Social Choice, Tilburg University Press: 125-139.

Brink, R. van den (2001) Banzhaf Permission Values for Games with a Permission Structure, Technical Report 341, Department of Mathematics, University of Texas at Arlington.

Brink, R. van den (2002) The Apex Power Measure for Directed Networks, Social Choice and Welfare 19: 845-867.

Brink, R. van den and Gilles, R.P. (1996) Axiomatizations of the Conjunctive Permission Value for Games with a Permission Structure, Games and Economic Behavior 12: 113-126.

Brink, R. van den and Gilles, R.P. (2000) Measuring Domination in Directed Graphs, Social Networks 22: 141-157.

Copeland, A.H. (1951) A Reasonable Social Welfare Function, mimeo, Seminar on Applications of Mathematics to Social Sciences, University of Michigan.

Daudi, P. (1986) Power in the Organization, Basil Blackwell.

Felsenthal, D.S. et al. (2003) In Defence of Voting Power Analysis: Responses to Albert, European Union Politics 4: 473-497.

Felsenthal, D.S. and Machover, M. (1998) The Measurement of Voting Power, Edward Elgar.

Felsenthal, D.S., Machover, M., and Zwicker, W.S. (1998) The Bicameral Postulate and Indices of A Priori Voting Power, Theory and Decision 44: 83-116.

Gilles, R.P., Owen, G., and Brink, R. van den (1992) Games with Permission Structures: The Conjunctive Approach, International Journal of Game Theory 20: 277-293.

Gilles, R.P. and Owen, G. (1994) Games with Permission Structures: The Disjunctive Approach, Mimeo, Department of Economics, Virginia Polytechnic Institute and State University.

Grofman, B. and Owen, G. (1982) A Game Theoretic Approach to Measuring Degree of Centrality in Social Networks, Social Networks 4: 213-224.

Harré, R. (1970) Powers, British Journal of the Philosophy of Science 21: 81-101.

Herings, P.J.-J., Laan, G. van der, and Talman, D. (2005) The Positional Power of Nodes in Digraphs, Social Choice and Welfare 24: 439-454.

Holler, M.J. et al. (eds) (2002) Jahrbuch für Neue Politische Ökonomie 20: Power and Fairness, Mohr Siebeck. 
Holler, M.J. and Owen, G. (eds) (2000) Power Measures, Vol. I, Homo Oeconomicus 17.

Holler, M.J. and Owen, G. (eds) (2001) Power Indices and Coalition Formation, Kluwer Academic Publishers.

Holler, M.J. and Owen, G. (eds) (2002) Power Measures, Vol. II, Homo Oeconomicus 19.

Holler, M.J. and Gambarelli, G. (eds) (2006) Power Measures, Vol. III, Homo Oeconomicus 23.

Hu, X. and Shapley, L.S. (2003) On Authority Distributions in Organisations: Controls, Games and Economic Behavior 45: 153-170.

Johnston, P. and Gill J. (1993) Management Control and Organizational Behaviour, Paul Chapman Publishing.

Kolpin, V.W. (1988) A Note on Tight Extensive Game Forms, International Journal of Game Theory 17: 187-191.

Kolpin, V.W. (1989) Core Implementation via Dynamic Games Forms, Social Choice and Welfare 6: 205-225.

Kuhn, H.W. (1953) Extensive Games and the Problem of Information: in H.W. Kuhn and A.W. Tucker (eds), Contributions to the Theory of Games II (Annals of Mathematics Studies 28), Princeton University Press, 193-216.

Mackenzie, K.D. (1976) A Theory of Group Structures, Vol. I: Basic Theory, Gordon and Breach Science Publishers.

Martin, J. (1998) Organizational Behaviour, International Thompson Business Press.

Mas-Collell, A., Whinston, M.D., and Green, J.R. (1995) Microeconomic Theory, Oxford University Press.

Miller, N.R. (1982), Power in Game Forms, in M.J. Holler (ed.), Power, Voting and Voting Power, Physica Verlag, 33-51.

Mizruchi, M.S. and Potts, B.B. (1998) Centrality and Power Revisited: Actor Success in Group Decision Making, Social Networks 20: 353-387.

Morriss, P. (1987/2002) Power: A Philosophical Analysis, Manchester University Press.

Radner, R. (1992) Hierarchy: The Economics of Managing, Journal of Economic Literature 30: $1382-1415$.

Russett, B.M. (1968) Probalism and the Number of Units: Measuring Influence Concentration, American Political Science Review 62: 476-480.

Selten, R. (1975) Reexamination of the Perfectness Concept for Equilibrium Points in Extensive Games, International Journal of Game Theory 4: 25-55.

Shapley, L.S. (1962) Simple Games: An Outline of the Descriptive Theory, Behavioral Science 7: 59-66.

Shapley, L.S. and Palamara, J.R. (2000a) Control Games and Organizations, UCLA Working Paper 795.

Shapley, L.S. and Palamara, J.R. (2000b) Simple Games and Authority Structure, UCLA Working Paper 796.

Shapley, L.S. and Shubik, M. (1954) A Method for Evaluating the Distribution of Power in a Committee System, American Political Science Review 48: 787-792.

Steffen, F. (2002) Essays in the Theory of Voting Power, PhD Dissertation, University of Hamburg.

Straffin, P.D. (1977) Homogeneity, Independence, and Power Indices, Public Choice 30: $107-118$.

Straffin, P.D. (1978) Probability Models for Power Indices, in: P.C. Ordeshook (ed.), Game Theory and Political Science, New York University Press, 477-510.

Taylor, A.D. and Zwicker, W.S. (1999) Simple Games, Princeton University Press. 